\title{
The Adult Mouse Hippocampal Progenitor Is Neurogenic But Not a Stem Cell
}

\author{
Natalie D. Bull and Perry F. Bartlett \\ Queensland Brain Institute, The University of Queensland, Brisbane QLD 4072, Australia
}

\begin{abstract}
The aim of this investigation was to characterize the proliferative precursor cells in the adult mouse hippocampal region. Given that a very large number of new hippocampal cells are generated over the lifetime of an animal, it is predicted that a neural stem cell is ultimately responsible for maintaining this genesis. Although it is generally accepted that a proliferative precursor resides within the hippocampus, contradictory reports exist regarding the classification of this cell. Is it a true stem cell or a more limited progenitor? Using a strict functional definition of a neural stem cell and a number of in vitro assays, we report that the resident hippocampal precursor is a progenitor capable of proliferation and multipotential differentiation but is unable to self-renew and thus proliferate indefinitely. Furthermore, the mitogen FGF-2 stimulates proliferation of these cells to a greater extent than epidermal growth factor (EGF). In addition, we found that BDNF was essential for the production of neurons from the hippocampal progenitor cells, being required during proliferation to trigger neuronal fate. In contrast, a bona fide neural stem cell was identified in the lateral wall of the lateral ventricle surrounding the hippocampus. Interestingly, EGF proved to be the stronger mitogenic factor for this cell, which was clearly a different precursor from the resident hippocampal progenitor. These results suggest that the stem cell ultimately responsible for adult hippocampal neurogenesis resides outside the hippocampus, producing progenitor cells that migrate into the neurogenic zones and proliferate to produce new neurons and glia.
\end{abstract}

Key words: hippocampus; progenitor; lateral ventricle; neural stem cell; BDNF; adult neurogenesis

\section{Introduction}

It is now clear that there is a continual turnover of hippocampal neurons throughout life (Altman and Das, 1965; Cameron et al., 1993; Palmer et al., 1995, 2000; Rietze et al., 2000; Seri et al., 2001; Garcia et al., 2004; Nyberg et al., 2005; Schmetsdorf et al., 2005). This neurogenesis appears to play a role in the maintenance of normal hippocampal function (Raber et al., 2004) and can be modulated by environmental factors (Gould et al., 1999; van Praag et al., 1999), antidepressants (Santarelli et al., 2003), and disease states (Nakatomi et al., 2002; Jin et al., 2004). However, it is still unclear whether a resident stem cell population underlies this proliferation, as has been demonstrated within the adult subependyma of the rostral lateral ventricle (Reynolds and Weiss, 1992; Morshead et al., 1994; Gritti et al., 1996; Weiss et al., 1996). A possible contributing factor to this debate is the differing application of the terms "stem cell," "progenitor," and "precursor," which are frequently and incorrectly used interchangeably, a habit that impacts negatively on the field, as highlighted by Reynolds and Rietze (2005). Here, we use the following functional definition of a stem cell: a proliferative cell with the capacity for

Received Aug. 3, 2005; revised Sept. 30, 2005; accepted 0ct. 2, 2005.

This work was supported by Australian National Health and Medical Research Council (NHMRC) Project Grant 301134 and an NHMRC Program Grant awarded to P.F.B. N.D.B. was supported by the Australasian Spinal Research Trust Perry Cross Fellowship, and P.F.B. was supported by an Australian Research Council Federation Fellowship. We thank Rowan Tweedale for proofreading this manuscript.

Correspondence should be addressed to Natalie D. Bull, Queensland Brain Institute, University of Queensland, Brisbane QLD 4072, Australia. E-mail: n.bull@uq.edu.au.

DOI:10.1523/JNEUROSCI.3249-05.2005

Copyright $\odot 2005$ Society for Neuroscience $\quad$ 0270-6474/05/2510815-07\$15.00/0 long-term (throughout the life of the animal) self-renewal that is multipotent, in this case generating the main classes of neural cell types (glia and neurons). In contrast, progenitor cells are more limited in their proliferative capacity, cannot self-renew, and may or may not be multipotent. The term precursor is reserved for an undefined proliferative cell.

Cells with the functional characteristics of neural stem cells (NSCs) have been isolated previously from the adult rat hippocampus (Palmer et al., 1997). It was demonstrated in vitro, using an adherent culture system with serum exposure, that these proliferative cells are fibroblast growth factor 2 (FGF-2) responsive, multipotent, and can be maintained in culture, suggesting long-term self-renewal. In contrast, the adult rodent dentate gyrus has been found to contain only restricted progenitor cells (Seaberg and van der Kooy, 2002). In this study, cells from the dentate gyrus proliferated in response to epidermal growth factor (EGF) plus FGF-2, differentiating into both neurons and glia but failing a test for self-renewal. Furthermore, self-renewal could not be attained using the adherent culture system used by Palmer et al. (1997), leading to the conclusion that the resident cells underlying rodent hippocampal neurogenesis are progenitors.

In the present study, we sought to clarify the nature of the proliferative population of cells residing within the adult murine hippocampus and nearby subependymal zone of the lateral wall of the lateral ventricle [here termed the posterior lateral ventricle $(\mathrm{pLV})$ ]. Initially, we used a clonal neurosphere assay to determine the frequency of proliferative hippocampal and pLV precursors. Neurospheres were assessed for precursor multipotentiality and 


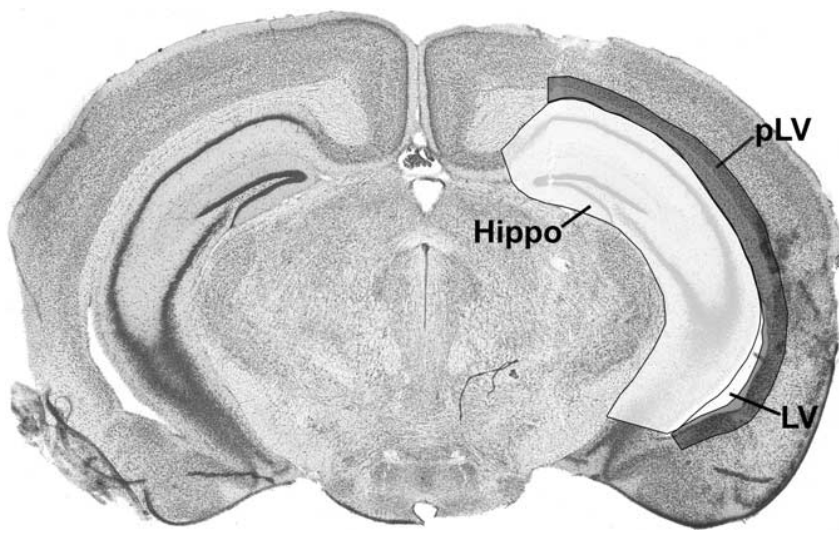

Figure 1. Diagram of primary tissue dissected for in vitro assay of stem-cell activity. A2-mmthick coronal slice was cut between -1.22 and $-2.70 \mathrm{~mm}$, relative to bregma, as determined from an adult mouse brain atlas (Paxinos and Franklin, 2001). The slice was placed caudal side up and the tissue dissected using two pairs of curved forceps. First, the midbrain was removed, and then the hippocampus, as a whole, was separated gently from the corpus callosum. Finally, the $\mathrm{pLV}$, including the corpus callosum, was pinched away from the cortex. Hippo, Hippocampus; LV, lateral ventricle. This image is from Paxinos and Franklin (2001) with permission.

passaged to assess the potential for self-renewal. In addition, we used the recently developed NeuroCult neural colony-forming cell assay (StemCell Technologies, Vancouver, British Columbia, Canada) (Reynolds et al., 2003; Adams et al., 2004) to distinguish between colonies of stem cell and progenitor cell origin.

\section{Materials and Methods}

Primary neurosphere cultures. Animals were treated in accordance with the Australian Code of Practice for the Care and Use of Animals for Scientific Purposes. All experiments were approved by the University of Queensland Animal Ethics Committee. Adult (6-8 weeks of age) male C57/BL6 mice were killed by cervical dislocation and their brains immediately removed. A 2-mm-thick coronal slice was cut between -1.22 and $-2.70 \mathrm{~mm}$, relative to bregma, as determined from an adult mouse brain atlas (Paxinos and Franklin, 2001). The slice was placed caudal side up and the tissue dissected using two pairs of curved forceps (for areas dissected, see Fig. 1). First, the midbrain was removed, and then the hippocampus, as a whole, was separated gently from the corpus callosum. Finally, the lateral wall of the lateral ventricle (or pLV), including the corpus callosum, was pinched away from the cortex. The tissue samples, which were processed in parallel, were diced with a scalpel blade and then digested enzymatically with $0.1 \%$ trypsin-EDTA (Invitrogen, San Diego, CA) for $7 \mathrm{~min}$ at $37^{\circ} \mathrm{C}$. The digestion was stopped by washing with $0.014 \% \mathrm{w} / \mathrm{v}$ trypsin inhibitor (type I-S from soybean; Sigma-Aldrich Australia, Sydney, Australia) dissolved in HEPES-buffered minimum essential medium (HEM), which consisted of minimum essential medium (Invitrogen) supplemented with 16 mM HEPES (Sigma-Aldrich Australia) and $100 \mathrm{U} / \mathrm{ml}$ penicillin/streptomycin (Invitrogen). The tissue was centrifuged at 100 relative centrifugal force ( $\mathrm{rcf}$ ) for $7 \mathrm{~min}$, after which the pellet was resuspended in $200 \mu \mathrm{l}$ of $0.1 \mathrm{~m}$ Dulbecco's PBS (calcium and magnesium free; Invitrogen) and mechanically triturated until smooth. The cells were washed with HEM and filtered through a $70 \mu \mathrm{m}$ cell sieve (BD Biosciences Australia, North Ryde, Australia). After centrifugation at $100 \mathrm{rcf}$ for $7 \mathrm{~min}$, they were resuspended in PBS and the number of viable cells counted on a hemocytometer using $0.08 \%$ trypan blue (Sigma-Aldrich Australia) in PBS. The cells were plated at a density of 7000 cells $/ \mathrm{cm}^{2}$ in six-well plates (BD Biosciences Australia) with $3 \mathrm{ml}$ of complete medium per well. Complete medium consisted of mouse NeuroCult NSC basal medium plus mouse NeuroCult NSC proliferation supplements (StemCell Technologies) with $2 \%$ bovine serum albumin (Roche, Basel, Switzerland) and $2 \mu \mathrm{g} / \mathrm{ml}$ heparin (Sigma-Aldrich Australia). The following growth factors were also included as specified: 20 $\mathrm{ng} / \mathrm{ml}$ purified mouse receptor-grade EGF (BD Biosciences Australia), 10 $\mathrm{ng} / \mathrm{ml}$ recombinant bovine FGF-2 (Roche), and $100 \mathrm{ng} / \mathrm{ml}$ recombinant human brain-derived neurotrophic factor (BDNF; Cytolab, Rehovot, Israel) unless otherwise stated. Primary hippocampal cells were incubated for $8 \mathrm{~d}$, and pLV cells for $7 \mathrm{~d}$, in humidified $5 \% \mathrm{CO}_{2}$ to permit neurosphere formation. The neurospheres were then counted and collected for either differentiation or passaging.

For the limiting dilution experiments, primary cells were plated at reducing densities in 96-well plates (BD Biosciences Australia) with 200 $\mu \mathrm{l}$ per well complete medium containing EGF and FGF-2. Hippocampal cells were plated at 40000, 20000, 10000, 7500, 5000, 2500, 1000, 500, 100, and 50 cells per well, whereas pLV tissue was plated at 10000, 5000, 1000, $750,500,250,100,50$, and 10 cells per well. After $7 \mathrm{~d}$ in vitro, the fraction of wells negative for neurosphere formation was quantified. These data were then log transformed and plotted against plating density. A linear regression was performed and a straight line passing through zero fitted to the data. The intercept of $\log (37 \%)$ gave the frequency of cells capable of proliferating to form a neurosphere [see Lefkovits (1979) for the theory underlying this procedure].

Fluorescence-activated cell sorting. Primary tissue was dissociated as described above, and the single-cell suspension was incubated for $15 \mathrm{~min}$ at room temperature in PBS containing the lectin peanut agglutinin (PNA) directly conjugated to fluorescein (1:200; Vector Laboratories, Burlingame, CA). The cells were then washed with PBS containing the vital dye DiI (Invitrogen), centrifuged at $100 \mathrm{rcf}$ for $7 \mathrm{~min}$, and resuspended in PBS in preparation for sorting. Unsorted, control cells were plated in complete medium at 3500 cells $/ \mathrm{cm}^{2}$ in 24 -well plates. Using a FACSVantage (Becton Dickinson, Melbourne, Australia) cell sorter, a gating strategy was applied to exclude excess debris (based on forward scatter) and dead cells (based on the uptake of DiI into damaged cells). Of the remaining "live" cells, PNA-negative cells were separated from PNApositive cells based on gates set around negative controls (cells incubated in parallel without PNA). The two populations of cells were collected into complete medium and plated into 96 -well plates (200 $\mu$ l per well). The cells were incubated in humidified $5 \% \mathrm{CO}_{2}$, and, $2 \mathrm{~h}$ after plating, the number of cells in five representative wells per plate were counted under an inverted microscope to estimate the number of cells collected per population. After $8 \mathrm{~d}$ of growth, the number of neurospheres produced by each population was quantified.

Neurosphere differentiation. Neurospheres were plated onto poly-Dlysine-coated BioCoat eight-well culture slides (BD Biosciences Australia) in NeuroCult NSC basal medium plus mouse NeuroCult NSC proliferation supplements without growth factors or serum. The neurospheres were allowed to differentiate for 5-7 d in humidified 5\% $\mathrm{CO}_{2}$ until flattened and adherent. The differentiated neurospheres were then fixed with $4 \%$ paraformaldehyde (Sigma-Aldrich Australia) in $0.1 \mathrm{M}$ PBS at room temperature for $30 \mathrm{~min}$. After washing with PBS, they were double stained for the neuronal marker $\beta$ III tubulin and the astrocytic marker glial fibrillary acidic protein (GFAP) with a $4^{\prime}, 6^{\prime}$-diamidino-2phenylindole (DAPI) counter stain (see below) or labeled for the oligodendrocyte marker $\mathrm{O} 4$.

Immunocytochemistry. The cells were incubated for $60 \mathrm{~min}$ at room temperature with blocking solution: $5 \%$ fetal bovine serum (JHR Biosciences, Brooklyn, Australia) plus 5\% normal goat serum (SigmaAldrich Australia) in 0.1 м PBS containing 0.1\% Triton X-100 (SigmaAldrich Australia). The blocking solution was replaced with fresh blocking solution containing either mouse monoclonal $\beta$ III tubulin antibody (1:2000; Promega, Madison, WI) plus rabbit polyclonal GFAP antibody (1:500; DakoCytomation, Carpinteria, CA) or mouse monoclonal $\mathrm{O} 4$ antibody (1:100; Chemicon, Boronia, Australia) and incubated for $60 \mathrm{~min}$ at room temperature. The cells were washed with PBS and incubated for $30 \mathrm{~min}$ at room temperature in blocking solution containing AlexaFluor 568 anti-mouse antibody (1:1000; Invitrogen), Alexafluor 488 anti-rabbit antibody (1:1000; Invitrogen), and DAPI (1:5000; Sigma-Aldrich Australia). After washing with PBS, the slides were coverslipped with fluorescence mounting medium (DakoCytomation) before viewing on a Zeiss (Gôttingen, Germany) upright fluorescence microscope. Images were captured by a digital camera linked to a computer running Axioscope version 4 (Zeiss).

Neurosphere passaging. Neurospheres were collected and centrifuged for $5 \mathrm{~min}$ at $100 \mathrm{rcf}$. They were then treated for $2 \mathrm{~min}$ at room tempera- 
ture with $0.1 \%$ trypsin-EDTA, followed by washing with trypsin inhibitor in HEM. The neurospheres were centrifuged for $5 \mathrm{~min}$ at $100 \mathrm{rcf}$, resuspended in PBS, and triturated mechanically until dissociated. Trypan blue staining was used to evaluate the number of cells, both viable and total number, on a hemocytometer. The passaged cells were then replated with complete medium at a density of $1 \times 10^{4} \mathrm{cells} / \mathrm{cm}^{2}$ in tissue-culture flasks (Nunc, Rochester, NY) or tissue-culture plates (BD Biosciences Australia), as appropriate. Hippocampal neurospheres were passaged every $8 \mathrm{~d}$, whereas pLV neurospheres were passaged every $7 \mathrm{~d}$.

Colony-forming assay. Primary tissue was dissociated and the number of viable cells counted as for the primary neurosphere cultures (see above). Cells were plated using the mouse NeuroCult neural colonyforming cell assay kit (StemCell Technologies) as per the manufacturer's instructions, except that they were plated at a density of $1 \times 10^{5}$ cells per $35 \mathrm{~mm}$ cell culture dish with $2 \mathrm{~mm}$ grid (Nunc). The cells were then incubated for $21 \mathrm{~d}$ in humidified $5 \% \mathrm{CO}_{2}$, and growth factors were added every $7 \mathrm{~d}$. After $21 \mathrm{~d}$ in vitro, the colony diameters were measured using an eyepiece graticule on an inverted Leica (Nussloch, Germany) light microscope with phase contrast.

\section{Results}

\section{The adult mouse hippocampus and pLV contain} proliferative cells

Cells were isolated from the entire hippocampus and the subependymal zone of the lateral wall of the lateral ventricle at the level of the hippocampus (here termed the pLV) and cultured in the neurosphere assay to enumerate precursor cell number. The neurosphere assay facilitates the proliferation of stem cells and/or progenitor cells in vitro such that individual cells divide over a period of $\sim 1$ week to produce spherical, detached colonies termed neurospheres, which are typically $50-140 \mu \mathrm{m}$ in diameter. It is important to remember that the generation of a neurosphere is not conclusive evidence for the presence of a stem cell, because both stem cells and progenitors are capable of proliferating to form neurospheres (Reynolds and Rietze, 2005). Both the adult mouse hippocampus and the pLV contained cells capable of forming neurospheres when cultured in the combined presence of the mitogens EGF and FGF-2 (Fig. 2). Use of a limiting dilution neurosphere assay, in which cells were plated at reducing concentrations and analyzed to determine the frequency of colonyforming cells, revealed that $\sim 1$ hippocampal cell in 9000 proliferated (Fig. 2A), whereas $\sim 1$ pLV cell in 750 formed a neurosphere (Fig. $2 B$ ). In addition, the limiting dilution neurosphere assay revealed that both cell types could proliferate at a clonal density and did not require the support of other cells. The neurospheres formed from the pLV tissue were reminiscent of neurospheres cultured from the rostral subventricular zone (SVZ), being on average $120 \mu \mathrm{m}$ in diameter after $7 \mathrm{~d}$ in vitro, whereas the hippocampal neurospheres were significantly smaller, only reaching $70-90 \mu \mathrm{m}$ in diameter after $8 \mathrm{~d}$ in vitro. Furthermore, hippocampal neurospheres did not increase in size even when left for up to 2 weeks in culture.

\section{The adult mouse hippocampus contains progenitor cells, whereas the pLV contains bona fide stem cells}

Isolated cells from the adult mouse hippocampus and pLV were cultured in the NeuroCult neural colony-forming cell assay, which has been developed to differentiate between colonies formed by bona fide neural stem cells as opposed to progenitor cells (Reynolds et al., 2003; Adams et al., 2004). This assay is similar to the neurosphere assay in that it uses a defined serumfree medium in which single cells proliferate to form clonal colonies; however, the colonies are cultured in a semisolid collagen matrix that permits them to be cultured for up to 3 weeks. In this assay, only the largest colonies ( $>1.5 \mathrm{~mm}$ in diameter) have been

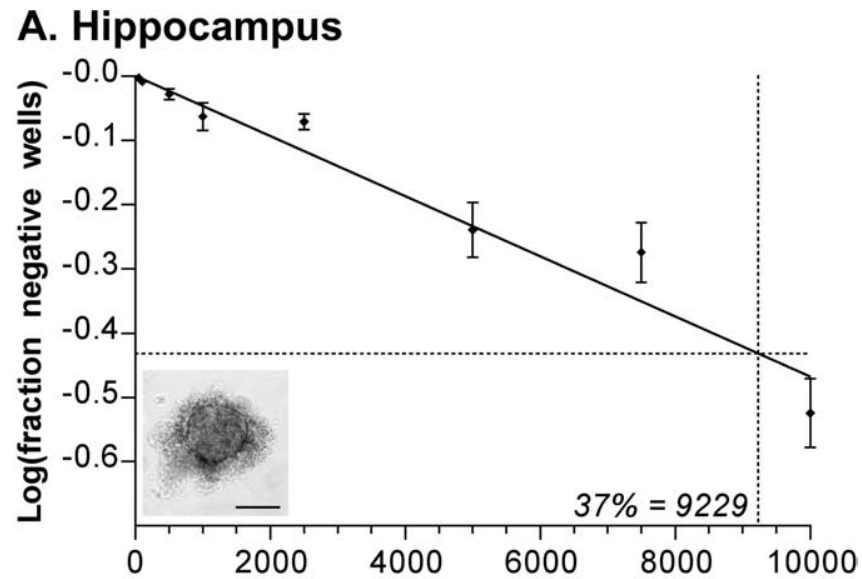

B. $\mathrm{pLV}$

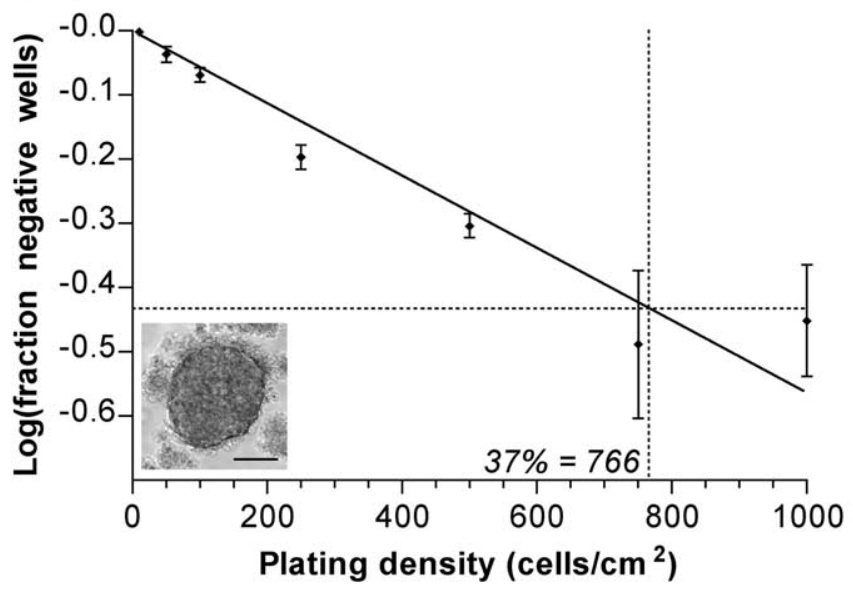

Figure 2. Proliferative cell frequency when primary tissue was cultured with both EGF and FGF-2.A, Primary hippocampal neurosphere limiting dilution assay $(n=9$; mean \pm SEM). The intercept of $\log (37 \%$ negative wells) gave a neurosphere-forming frequency of one neurosphere for every 9229 cells plated. Inset, Representative photograph of a primary hippocampal neurosphere. Scale bar, $100 \mu \mathrm{m}$. B, Primary pLV neurosphere limiting dilution assay $(n=6$; mean $\pm S E M)$. Neurosphere-forming frequency was calculated as one neurosphere for every 766 cells plated. Inset, Representative photograph of a primary pLV neurosphere. Scale bar, $100 \mu \mathrm{m}$.

shown to originate from a stem cell, based on the generation of long-term neurosphere cultures from individual colonies, whereas small colonies are formed by more limited progenitors (Reynolds et al., 2003; Adams et al., 2004). When grown in the presence of EGF and FGF-2, proliferative cells from the hippocampus only formed small colonies ( $\leq 1.0 \mathrm{~mm}$ diameter) (Fig. $3 A$ ), indicating the absence of a stem-cell population. In contrast, pLV cells produced a range of colony sizes, with a small percentage $>1.5 \mathrm{~mm}$ in diameter, similar to colonies formed from adult rostral SVZ cells (Adams et al., 2004).

Neurospheres from both the hippocampus and the pLV were also passaged repeatedly, as bulk cultures, to determine whether they could be maintained in culture through a large number of passages. Given that progenitor cells do not exhibit long-term self-renewal, only cultures containing stem cells can be expanded continuously, whereas cultures based on progenitor cells gradually die out. The neurospheres grown from pLV cells were easily maintained and expanded in culture for up to at least 10 passages (Fig. $3 B$ ). In contrast, long-term cultures could not be established from hippocampal neurospheres (Fig. $3 B$ ). At each passage, the number of hippocampal neurospheres diminished until, after the 
third passage, no additional neurospheres were generated. The neurospheres generated from the hippocampal cultures were always small, being $<100 \mu \mathrm{m}$ in diameter

\section{Hippocampal progenitors are more} responsive to FGF-2, whereas pLV stem cells are primarily EGF responsive Cells from both tissue types were cultured in the presence of either EGF or FGF-2, or both growth factors together, to determine whether proliferation was differentially controlled by the mitogens. When cultured in the colony-forming assay in the presence of EGF alone, proliferative hippocampal cells divided less often and generated only very small $(<200 \mu \mathrm{m})$ colonies compared with cultures generated when both growth factors were present, conditions that produced colonies up to $\sim 500 \mu \mathrm{m}$ in diameter (Fig. $4 A$ ). In contrast, when cultured with FGF-2 alone, hippocampal progenitors proliferated to a similar extent to that observed when both mitogens were included in the assay and produced colonies of $\sim 500 \mu \mathrm{m}$ in diameter (Fig. 4A). Although EGF stimulated hippocampal progenitor division to a lesser extent than FGF-2, the number of cells capable of forming a neurosphere in either EGF or FGF-2 did not differ significantly (data not shown).

In contrast to the hippocampal progenitor, cells from the pLV cultured with EGF alone proliferated to a similar extent to that observed in the presence of both mitogens (Fig. $4 \mathrm{~B}$ ) and generated a wide range of colony sizes, including large colonies derived from stem cell proliferation. In contrast, the culture of pLV tissue in FGF-2 alone induced limited proliferation and the production of only small colonies, with none larger than $1 \mathrm{~mm}$ in diameter (Fig. 4B). As observed with the hippocampal progenitor cells, culture with the individual mitogens did not alter the number of cells capable of forming a neurosphere (data not shown), just the extent of proliferation.

\section{Sorting for PNA-negative cells enriches for both pLV stem cells and hippocampal progenitors}

We investigated whether, as demonstrated previously for the rostral SVZ stem cell (Rietze et al., 2001), precursor cells from the hippocampus and pLV could be enriched using fluorescenceactivated cell sorting (FACS) based on negative binding of the lectin PNA. For both primary hippocampal and pLV tissue, FACS analysis was used to separate and collect the cells that did not bind the lectin PNA ( $\mathrm{PNA}^{-}$population) from those that did $\left(\mathrm{PNA}^{+}\right.$ population). The sorted cells were then cultured in the neurosphere assay to determine proliferative activity. Sorting based on the negative binding of PNA enriched for neurosphere activity in both tissue types. In the case of the hippocampus, one cell in $563 \pm 163$ (mean \pm SEM; $n=3$ ) proliferated to form a neurosphere, which was enriched compared with one cell in $9167 \pm$ 1093 (mean \pm SEM; $n=3$ ) for parallel, unsorted control samples. Similarly, one pLV PNA ${ }^{-}$cell in $127 \pm 19$ (mean \pm SEM;
B.

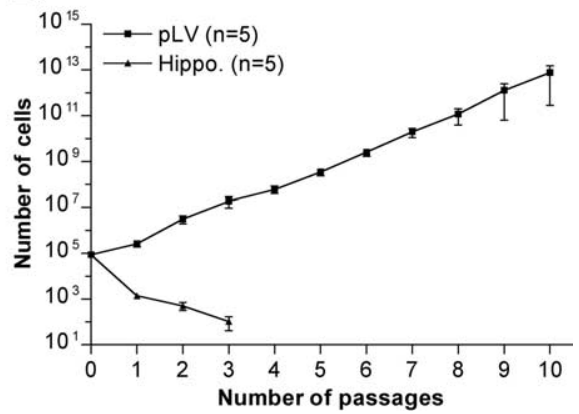

Figure 3. Potential for long-term proliferation in vitro in the presence of both EGF and FGF-2. $\boldsymbol{A}$, Colony diameters (in millime-

A. Hippocampus

Differential effects of EGF and FGF-2 on proliferation. $\boldsymbol{A}$, Colony diameters (in micrometers) produced by primary cell assay (mean \pm SEM). $\boldsymbol{B}$, Colony diameters (in millimeters) when primary $\mathrm{pLV}$ cells were cultured in the presence of EGF plus FGF-2 or each growth factor alone in the neural colony-forming cell assay (mean $\pm \mathrm{SEM}$ ).

$n=3)$ proliferated, compared with one in every $722 \pm 24$ (mean $\pm \mathrm{SEM} ; n=3$ ) in unsorted control samples.

\section{BDNF is essential for hippocampal progenitor cells to} generate neurons

Primary neurospheres from both the hippocampus and the pLV were differentiated, by removal of mitogens and provision of an adherent substrate, and double labeled for the neuronal marker $\beta$ III tubulin and the astrocytic marker GFAP or a single label for the oligodendrocyte marker O4. Serum was not added to the differentiation medium, because this was found to reduce the number of neurons generated (our unpublished observation). When neurospheres were grown in EGF and FGF-2 and then differentiated, pLV neurospheres were found to be multipotent, producing neurons, astrocytes, and oligodendrocytes (Fig. 5A). In contrast, under these growth conditions, the hippocampal neurospheres only generated glia, producing astrocytes and oligodendrocytes (Fig. 5B) but no neurons. However, when hippocampal neurospheres were cultured in complete medium containing BDNF, approximately one-half were capable of producing both neurons and glia after differentiation (Fig. 5C). Neither tissue produced neurospheres that solely generated neurons. When added at different stages during neurosphere growth and included during differentiation, BDNF was found to stimulate neurogenesis when it was present from the start of proliferation (day 0 in vitro) or when added after $5 \mathrm{~d}$ in vitro (for an additional 3 d proliferation). However, when BDNF was added only during neurosphere differentiation, it was incapable of stim- 

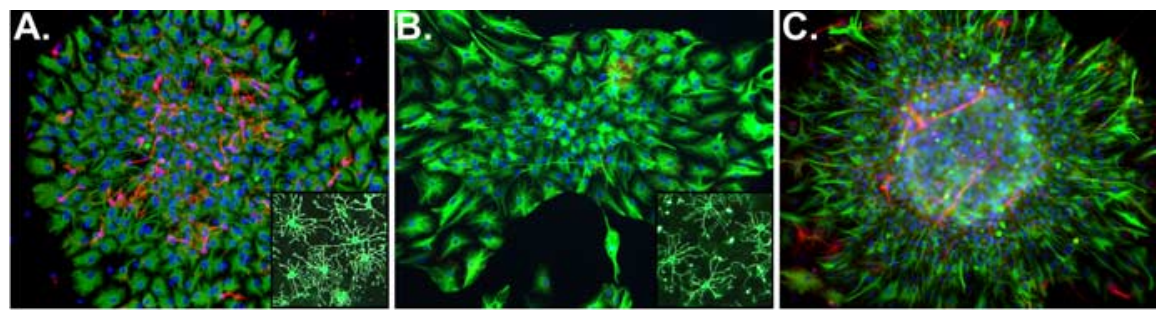

D.

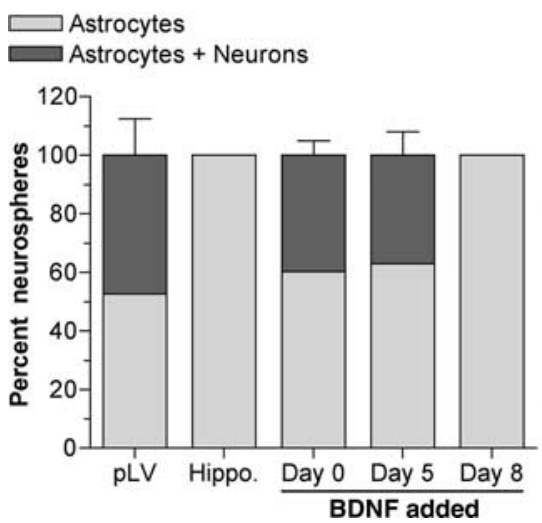

E.

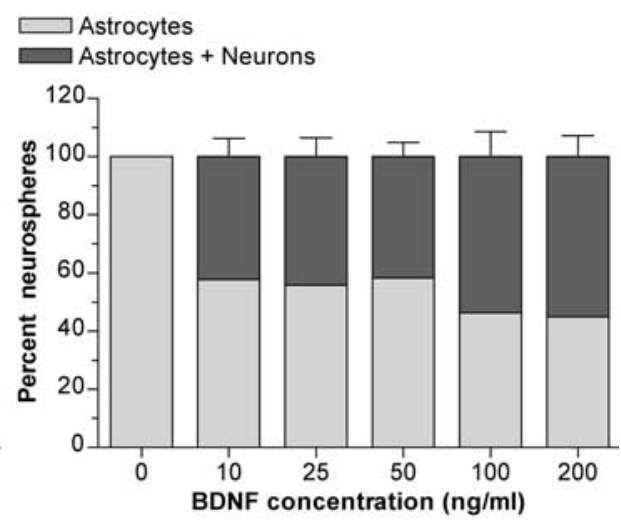

Figure 5. Differentiation of primary neurospheres. $A-C$, Representative differentiated neurospheres immunolabeled for GFAP (green) and $\beta I I I$ tubulin (red) and counterstained with DAPI (blue). Primary pLV neurosphere $(\boldsymbol{A})$, primary hippocampal neurosphere grown in the presence of EGF and FGF-2 $(\boldsymbol{B})$, and a primary hippocampal neurosphere cultured in the presence of EGF, FGF-2, and BDNF (100 ng/ml) ( $(C$ ) are shown. $\boldsymbol{A}, \boldsymbol{B}$, Insets, Immunolabeling for the oligodendrocyte marker 04 (green) in representative differentiated pLV $(\boldsymbol{A})$ and hippocampal $(\boldsymbol{B})$ neurospheres. $\boldsymbol{D}$, Percentage of differentiated neurospheres containing cells immunopositive for either GFAP alone (astrocytes) or GFAP plus $\beta$ III tubulin (neurons) after growth in the presence of EGF and FGF-2 or with BDNF (100 ng/ml) added to hippocampal cultures at different time points during neurosphere proliferation $(n=3$; mean $\pm S E M)$. $\boldsymbol{E}$, Percentage of differentiated neurospheres containing astrocytes alone or astrocytes plus neurons when primary hippocampal cells were cultured in increasing concentrations of BDNF $(n=3$; mean \pm SEM). Hippo., Hippocampus.

A.

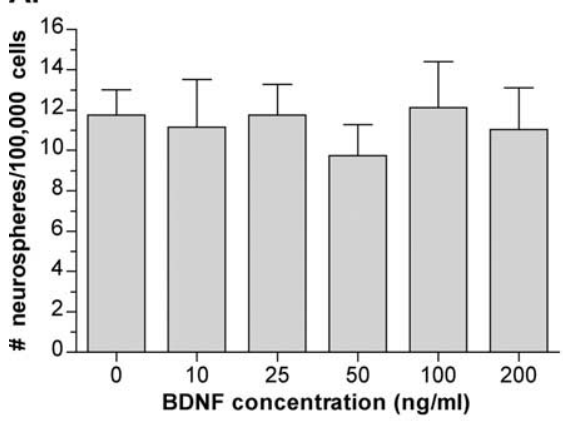

B.

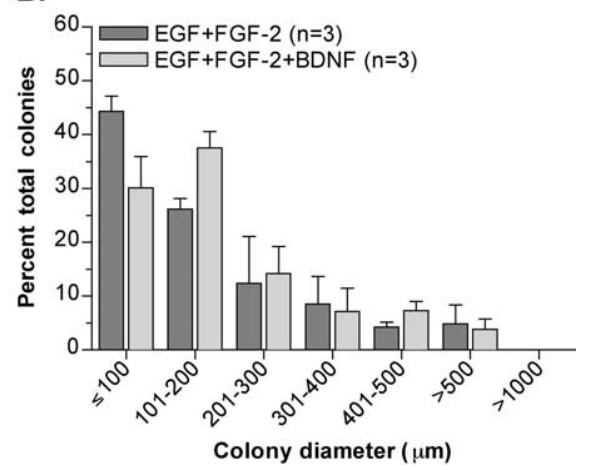

Figure 6. Effect of BDNF on hippocampal neurosphere frequency and proliferative potential. $\boldsymbol{A}$, Primary hippocampal neurosphere frequency when cells were cultured in the presence of EGF and FGF-2 plus BDNF at increasing concentrations ( $n=4$; mean \pm SEM). $\boldsymbol{B}$, Colony diameters (in micrometers) when primary hippocampal cells were cultured in the neural colony-forming cell assay in the presence of EGF and FGF-2 or EGF and FGF-2 plus BDNF (100 ng/ml). Values are mean \pm SEM.

ulating neuronal production (Fig. 5D). In addition, BDNF was found to stimulate neurogenesis at doses as low as $10 \mathrm{ng} / \mathrm{ml}$ when added to the culture medium from day 0 and included during differentiation (Fig. 5E).

\section{BDNF does not affect hippocampal progenitor proliferation or self-renewal}

Hippocampal cells were also cultured in both the NeuroCult neural colony-forming cell assay and the neurosphere assay, in the combined presence of BDNF, EGF, and FGF-2, to determine whether BDNF influences hippocampal progenitor proliferation or self-renewal. Including BDNF in the neurosphere culture medium, at doses from 10 to $200 \mathrm{ng} /$ $\mathrm{ml}$, did not change the number of hippocampal cells capable of generating a neurosphere (Fig. $6 A$ ). In addition, inclusion of BDNF $(100 \mathrm{ng} / \mathrm{ml})$ in the growth medium did not enable hippocampal neurospheres to be maintained through repeated passages in culture (data not shown). Furthermore, the addition of BDNF (100 ng/ml) did not alter the size of colonies generated from proliferative hippocampal cells (Fig. $6 \mathrm{~B}$ ) when compared with colonies grown in the presence of EGF and FGF-2, with no colonies larger than $1 \mathrm{~mm}$ diameter observed in either growth condition.

\section{Discussion}

The results presented here confirm the presence of a cell within the adult hippocampus that can proliferate to form clonal colonies in vitro. Furthermore, this cell is not reliant on support cells or factors released by cocultured cells to proliferate and can form small neurospheres under clonal conditions. However, although this cell is capable of generating new neurons and glia, it is unable to self-renew and proliferate over an extended period, as determined using the neurosphere assay. Although the hippocampal progenitor did produce some secondary and tertiary neurospheres, the number produced at each passage diminished until the culture died out. These data demonstrate that the proliferative cell located within the adult mouse hippocampus is a progenitor cell and not a bona fide stem cell, which by definition is required to be an extensively proliferating, multipotent cell capable of continual self-renewal. In support of this conclusion, use of the NeuroCult neural colony-forming cell assay revealed that the resident hippocampal precursor possessed only limited proliferative and selfrenewal capacity. The colony-forming assay has been shown previously to distinguish between colonies formed from extensively proliferating stem cells and more restricted progenitors based on the extent of proliferation over a 3 week period and subsequent colony size, with only the very largest colonies $(<1.5 \mathrm{~mm}$ diameter) being found to originate from a stem cell (Reynolds et al., 2003; Adams et al., 2004). Our conclusion that the hippocampus contains a proliferating progenitor population rather than a stem cell population contradicts the original classification by Palmer et al. (1997), who found an adult hippocampal cell with the functional characteristics of a stem cell. However, our conclusion lends support to that reached by Seaberg and van der Kooy (2002), who, using microdissection 
and the neurosphere assay, reported that the rodent dentate gyrus, the region of the hippocampus where new cells are born (Altman and Das, 1965; Cameron et al., 1993; Palmer et al., 2000), contains only progenitor cells that produce small neurospheres. It is possible that the conflicting nature of these findings may be attributable to pLV stem cell contamination of hippocampal samples used for the original classification (Palmer et al., 1997), although it is also possible that the different in vitro culture conditions used supported the growth of different populations of cells. Nevertheless, it must be noted that we attempted to culture hippocampal progenitors in the conditions detailed by Palmer et al. (1997) but failed to generate a culture, as also reported by Seaberg and van der Kooy (2002). In addition to different culture conditions, the original classification of hippocampal precursors was performed with rat tissue, whereas we used mice, which may also contribute to the contradictory findings.

Compared with the hippocampal progenitor, we found a cell within the surrounding lateral wall of the lateral ventricle that met stem cell classification. This proliferative cell was both multipotent and capable of self-renewal. Moreover, pLV cells generated a small percentage of very large colonies when cultured in the colony-forming assay, reminiscent of the profile produced by the traditional rostral SVZ stem cell (Adams et al., 2004). These results are supported partially by a previous report that cells from this region are capable of generating secondary neurospheres (Seaberg and van der Kooy, 2002). However, this assay was not carried through enough passages to be conclusive, given that progenitors may generate secondary neurospheres (as observed here), and it is only through long-term culture that self-renewal can be determined. In addition, we found that the pLV stem cell proliferates to a much greater extent when EGF is present in the culture medium than when only FGF- 2 is provided. Indeed, only small colonies ( $<1 \mathrm{~mm}$ in diameter) were generated when FGF-2 was the sole mitogen, compared with cultures containing either EGF alone or EGF plus FGF-2, which produced a wide range of colony sizes including very large colonies from extensive stem cell proliferation. Given that the pLV contains a mixed population of proliferative cells, a small percentage of which are stem cells, it is also possible that EGF controls stem cell division, whereas the progenitor population is responsive to both mitogens. Differential effects of EGF and FGF-2 on the proliferation of stem cells have been observed previously (Kuhn et al., 1997), where EGF was found to increase the production of astrocytes from rostral SVZ stem cells, but FGF-2 stimulated olfactory neuron production.

Similar experiments with cells isolated from the hippocampus revealed that FGF-2, rather than EGF, provides the stronger mitogenic signal for hippocampal progenitors. When cultured in the colony-forming assay in the presence of individual growth factors, hippocampal progenitors proliferated to a much greater extent when exposed to FGF-2, compared with EGF alone, and generated colonies of a similar size to those produced when both mitogens were present. This demonstrated that FGF-2 is important for controlling hippocampal progenitor division. Indeed, FGF-2 has been identified previously as playing a central role in signaling for hippocampal neurogenesis in normal rodents (Cheng et al., 2002), aged mice (Jin et al., 2003), and after traumatic brain injury (Yoshimura et al., 2001).

Evidence is rapidly accumulating to support a key role for BDNF in brain plasticity; it has been found to encourage neurogenesis and cell survival as well as to contribute to synaptic plasticity (for review, see Mattson et al., 2004). BDNF is implicated in maintaining basal levels of hippocampal neurogenesis (Lee et al.,
2002), stimulates neurogenesis (Scharfman et al., 2005), is upregulated in the dentate gyrus in response to neurogenic stimulating exercise (Farmer et al., 2004), and has a role in mediating the effect of antidepressant drugs on hippocampal neurogenesis (Sairanen et al., 2005). Not surprisingly, we also found that BDNF is critical for hippocampal neurogenesis. Only when BDNF was included in the growth medium during neurosphere proliferation were neurons produced by hippocampal progenitors. When hippocampal progenitors were grown in the presence of mitogens alone, only glia were generated, confirming previous results (Seaberg and van der Kooy, 2002). In fact, a BDNF concentration as low as $10 \mathrm{ng} / \mathrm{ml}$ induced neurogenesis, indicating a high sensitivity of the hippocampal progenitor to this neurotrophin. Interestingly, including BDNF alone during differentiation of neurospheres failed to produce neurons, suggesting that BDNF signaling during cell division influences the fate of some hippocampal progenitors toward the neuronal lineage. This conclusion is supported by the fact that BDNF is intimately tied to hippocampal neurogenesis in vivo. In addition, BDNF was found to have no role in stimulating or promoting hippocampal proliferation or self-renewal. It neither increased the number of hippocampal neurospheres generated nor enhanced the ability of hippocampal neurospheres to be passaged repeatedly. Furthermore, BDNF did not stimulate the growth of large hippocampal colonies in the colony-forming assay.

Here, we present strong evidence that the adult hippocampus contains progenitor cells that can proliferate, albeit to a limited extent, and produce new neurons. Given the large continuous turnover of hippocampal neurons, this raises an important question: where does the stem cell, which supports the supply of hippocampal progenitors responsible for the observed proliferation in the dentate gyrus and the production of new hippocampal neurons, reside? Although this study does not provide a definitive answer to this question, we have identified the presence of a stem cell population within the surrounding wall of the lateral ventricle that, given its close proximity, may provide a source for the hippocampal progenitors. Furthermore, FACS analysis suggested that the pLV stem cell and hippocampal progenitor may be related because both could be enriched based on negative binding of the lectin PNA, similar to that reported for the rostral SVZ stem cell (Rietze et al., 2001). It may be that the pLV stem cells produce a small number of progenitor cells that migrate into the dentate gyrus, where they proliferate to generate new hippocampal cells in much the same manner that cells within the rostral SVZ divide to supply the rostral migratory stream, leading to the production of new neurons within the olfactory bulb. In support of this theory, Nakatomi et al. (2002) observed cells migrating from the lateral ventricle into the hippocampal CA1 region after a stroke lesion. Moreover, the recovery of proliferation in the hippocampus, after mitotic suppression, lags behind that of the periventricular zone, which may be because of the time it takes new progenitor cells to migrate into the dentate gyrus (Becq et al., 2005). The identity of the stem cell underlying hippocampal cell turnover is of great importance, because hippocampal neurogenesis is known to decrease with age (Lichtenwalner et al., 2001; Jin et al., 2003; Heine et al., 2004) and impair spatial memory (Drapeau et al., 2003), while disruption of hippocampal neurogenesis leads to a reduction in cognitive function (Shors et al., 2001; Raber et al., 2004). Furthermore, this cell provides the basis for a possible intrinsic repair mechanism in response to injury (Liu et al., 1998; Jin et al., 2001, 2004; Nakatomi et al., 2002), such that its manipulation could lead to therapeutic outcomes. 


\section{References}

Adams DJ, Reynolds BA, Louis SA, Rietze RL (2004) The neurosphere assay overestimates the frequency of adult neural stem cells. Soc Neurosci Abstr 30:383.6.

Altman J, Das GD (1965) Autoradiographic and histological evidence of postnatal hippocampal neurogenesis in rats. J Comp Neurol 124:319-335.

Becq H, Jorquera I, Ben-Ari Y, Weiss S, Represa A (2005) Differential properties of dentate gyrus and CAl neural precursors. J Neurobiol 62:243-261.

Cameron HA, Woolley CS, McEwen BS, Gould E (1993) Differentiation of newly born neurons and glia in the dentate gyrus of the adult rat. Neuroscience 56:337-344.

Cheng Y, Black IB, DiCicco-Bloom E (2002) Hippocampal granule neuron production and population size are regulated by levels of bFGF. Eur J Neurosci 15:3-12.

Drapeau E, Mayo W, Aurousseau C, Le Moal M, Piazza PV, Abrous DN (2003) Spatial memory performances of aged rats in the water maze predict levels of hippocampal neurogenesis. Proc Natl Acad Sci USA 100:14385-14390.

Farmer J, Zhao X, van Praag H, Wodtke K, Gage FH, Christie BR (2004) Effects of voluntary exercise on synaptic plasticity and gene expression in the dentate gyrus of adult male Sprague-Dawley rats in vivo. Neuroscience 124:71-79.

Garcia A, Steiner B, Kronenberg G, Bick-Sander A, Kempermann G (2004) Age-dependent expression of glucocorticoid- and mineralocorticoid receptors on neural precursor cell populations in the adult murine hippocampus. Aging Cell 3:363-371.

Gould E, Beylin A, Tanapat P, Reeves A, Shors TJ (1999) Learning enhances adult neurogenesis in the hippocampal formation. Nat Neurosci 2:260-265

Gritti A, Parati EA, Cova L, Frolichsthal P, Galli R, Wanke E, Faravelli L, Morassutti DJ, Roisen F, Nickel DD, Vescovi AL (1996) Multipotential stem cells from the adult mouse brain proliferate and self-renew in response to basic fibroblast growth factor. J Neurosci 16:1091-1100.

Heine VM, Maslam S, Joels M, Lucassen PJ (2004) Prominent decline of newborn cell proliferation, differentiation, and apoptosis in the aging dentate gyrus, in absence of an age-related hypothalamus-pituitaryadrenal axis activation. Neurobiol Aging 25:361-375.

Jin K, Minami M, Lan JQ, Mao XO, Batteur S, Simon RP, Greenberg DA (2001) Neurogenesis in dentate subgranular zone and rostral subventricular zone after focal cerebral ischemia in the rat. Proc Natl Acad Sci USA 98:4710-4715.

Jin K, Sun Y, Xie L, Batteur S, Mao XO, Smelick C, Logvinova A, Greenberg DA (2003) Neurogenesis and aging: FGF-2 and HB-EGF restore neurogenesis in hippocampus and subventricular zone of aged mice. Aging Cell 2:175-183.

Jin K, Peel AL, Mao XO, Xie L, Cottrell BA, Henshall DC, Greenberg DA (2004) Increased hippocampal neurogenesis in Alzheimer's disease. Proc Natl Acad Sci USA 101:343-347.

Kuhn HG, Winkler J, Kempermann G, Thal LJ, Gage FH (1997) Epidermal growth factor and fibroblast growth factor-2 have different effects on neural progenitors in the adult rat brain. J Neurosci 17:5820-5829.

Lee J, Duan W, Mattson MP (2002) Evidence that brain-derived neurotrophic factor is required for basal neurogenesis and mediates, in part, the enhancement of neurogenesis by dietary restriction in the hippocampus of adult mice. J Neurochem 82:1367-1375.

Lefkovits I (1979) Limiting dilution analysis of cells in the immune system. In: Cells in the immune system, pp 8-10. Cambridge: Cambridge UP.

Lichtenwalner RJ, Forbes ME, Bennett SA, Lynch CD, Sonntag WE, Riddle DR (2001) Intracerebroventricular infusion of insulin-like growth factor-I ameliorates the age-related decline in hippocampal neurogenesis. Neuroscience 107:603-613.

Liu J, Solway K, Messing RO, Sharp FR (1998) Increased neurogenesis in the dentate gyrus after transient global ischemia in gerbils. J Neurosci 18:7768-7778.

Mattson MP, Maudsley S, Martin B (2004) BDNF and 5-HT: a dynamic duo in age-related neuronal plasticity and neurodegenerative disorders. Trends Neurosci 27:589-594.

Morshead CM, Reynolds BA, Craig CG, McBurney MW, Staines WA, Morassutti D, Weiss S, van der Kooy D (1994) Neural stem cells in the adult mammalian forebrain: a relatively quiescent subpopulation of subependymal cells. Neuron 13:1071-1082.

Nakatomi H, Kuriu T, Okabe S, Yamamoto S, Hatano O, Kawahara N, Tamura A, Kirino T, Nakafuku M (2002) Regeneration of hippocampal pyramidal neurons after ischemic brain injury by recruitment of endogenous neural progenitors. Cell 110:429-441.

Nyberg J, Anderson MF, Meister B, Alborn AM, Strom AK, Brederlau A, Illerskog AC, Nilsson O, Kieffer TJ, Hietala MA, Ricksten A, Eriksson PS (2005) Glucose-dependent insulinotropic polypeptide is expressed in adult hippocampus and induces progenitor cell proliferation. J Neurosci 25:1816-1825.

Palmer TD, Ray J, Gage FH (1995) FGF-2-responsive neuronal progenitors reside in proliferative and quiescent regions of the adult rodent brain. Mol Cell Neurosci 6:474-486.

Palmer TD, Takahashi J, Gage FH (1997) The adult rat hippocampus contains primordial neural stem cells. Mol Cell Neurosci 8:389-404.

Palmer TD, Willhoite AR, Gage FH (2000) Vascular niche for adult hippocampal neurogenesis. J Comp Neurol 425:479-494.

Paxinos G, Franklin KBJ (2001) The mouse brain in stereotaxic coordinates, Ed 2. London: Academic.

Raber J, Rola R, LeFevour A, Morhardt D, Curley J, Mizumatsu S, VandenBerg SR, Fike JR (2004) Radiation-induced cognitive impairments are associated with changes in indicators of hippocampal neurogenesis. Radiat Res 162:39-47.

Reynolds BA, Rietze RL (2005) Neural stem cells and neurospheres-reevaluating the relationship. Nat Methods 2:333-336.

Reynolds BA, Weiss S (1992) Generation of neurons and astrocytes from isolated cells of the adult mammalian central nervous system. Science 255:1707-1710.

Reynolds BA, Wagey R, Thomas TE, Eaves AC, Louis SA (2003) An in vitro colony assay which identifies neural stem cells with high and low proliferative potential. Soc Neurosci Abstr 29:124.5.

Rietze R, Poulin P, Weiss S (2000) Mitotically active cells that generate neurons and astrocytes are present in multiple regions of the adult mouse hippocampus. J Comp Neurol 424:397-408.

Rietze RL, Valcanis H, Brooker GF, Thomas T, Voss AK, Bartlett PF (2001) Purification of a pluripotent neural stem cell from the adult mouse brain. Nature 412:736-739.

Sairanen M, Lucas G, Ernfors P, Castren M, Castren E (2005) Brain-derived neurotrophic factor and antidepressant drugs have different but coordinated effects on neuronal turnover, proliferation, and survival in the adult dentate gyrus. J Neurosci 25:1089-1094.

Santarelli L, Saxe M, Gross C, Surget A, Battaglia F, Dulawa S, Weisstaub N, Lee J, Duman R, Arancio O, Belzung C, Hen R (2003) Requirement of hippocampal neurogenesis for the behavioral effects of antidepressants. Science 301:805-809.

Scharfman H, Goodman J, Macleod A, Phani S, Antonelli C, Croll S (2005) Increased neurogenesis and the ectopic granule cells after intrahippocampal BDNF infusion in adult rats. Exp Neurol 192:348-356.

Schmetsdorf S, Gartner U, Arendt T (2005) Expression of cell cycle-related proteins in developing and adult mouse hippocampus. Int J Dev Neurosci 23:101-112.

Seaberg RM, van der Kooy D (2002) Adult rodent neurogenic regions: the ventricular subependyma contains neural stem cells, but the dentate gyrus contains restricted progenitors. J Neurosci 22:1784-1793.

Seri B, Garcia-Verdugo JM, McEwen BS, Alvarez-Buylla A (2001) Astrocytes give rise to new neurons in the adult mammalian hippocampus. J Neurosci 21:7153-7160.

Shors TJ, Miesegaes G, Beylin A, Zhao M, Rydel T, Gould E (2001) Neurogenesis in the adult is involved in the formation of trace memories. Nature 410:372-376.

van Praag H, Kempermann G, Gage FH (1999) Running increases cell proliferation and neurogenesis in the adult mouse dentate gyrus. Nat Neurosci 2:266-270.

Weiss S, Dunne C, Hewson J, Wohl C, Wheatley M, Peterson AC, Reynolds BA (1996) Multipotent CNS stem cells are present in the adult mammalian spinal cord and ventricular neuroaxis. J Neurosci 16:7599-7609.

Yoshimura S, Takagi Y, Harada J, Teramoto T, Thomas SS, Waeber C, Bakowska JC, Breakefield XO, Moskowitz MA (2001) FGF-2 regulation of neurogenesis in adult hippocampus after brain injury. Proc Natl Acad Sci USA 98:5874-5879. 